\title{
Vital Roles of $\beta$-catenin in Trans-differentiation of Chondrocytes to Bone Cells
}

\author{
Yan Jing ${ }^{\bowtie \#}$, Junjun Jing ${ }^{3 \#, ~ K e ~ W a n g}{ }^{2}$, Kevin Chan², Stephen E. Harris ${ }^{4}$, Robert J. Hinton², Jian Q. Feng ${ }^{2 \bowtie}$ \\ 1. Department of Orthodontics, Texas A\&M University College of Dentistry, Dallas, TX, 75246, USA \\ 2. Department of Biomedical Sciences, Texas A\&M University College of Dentistry, Dallas, TX, 75246, USA \\ 3. State Key Laboratory of Oral Diseases, West China Hospital of Stomatology, Sichuan University, Chengdu, China, 610041. \\ 4. Department of Periodontics, University of Texas Health Science Center at San Antonio, San Antonio, TX, 78229, USA \\ \# Both authors contribute equally to this work. \\ $\triangle$ Corresponding authors: Yan Jing: yjing@tamhsc.edu; Department of Orthodontics, Texas A\&M University College of Dentistry, Dallas, TX, 75246, USA. \\ +1-214-370-7237 and Jian Q. Feng: jfeng@tamhsc.edu; Department of Biomedical Sciences, Texas A\&M University College of Dentistry, Dallas, TX, 75246, USA. \\ $+1-214-370-7235$ \\ (C) Ivyspring International Publisher. This is an open access article distributed under the terms of the Creative Commons Attribution (CC BY-NC) license \\ (https://creativecommons.org/licenses/by-nc/4.0/). See http://ivyspring.com/terms for full terms and conditions.
}

Received: 2017.10.04; Accepted: 2017.10.14; Published: 2018.01.01

\begin{abstract}
A recent breakthrough showing that direct trans-differentiation of chondrocytes into bone cells commonly occurs during endochondral bone formation in the growth plate, articular cartilage, and mandibular condylar cartilage suggests that chondrogenesis and osteogenesis are likely one continuous biological process instead of two separate processes. Yet, gene regulation of this cell transformation is largely unclear. Here, we employed cartilage-specific $\beta$-catenin loss-of-function $\left(\beta\right.$-catenin ${ }^{f \times / f x}$ ) and gain-of-function $\left(\beta\right.$-catenin $\left.{ }^{f \times(e x o n 3) / f \times(e x o n 3)}\right)$ models in the $R 26 R^{\text {Tomato }}$ background (for better tracing the cell fate of chondrocytes) to study the role of $\beta$-catenin in cell trans-differentiation. Using histological, immunohistochemical, and radiological methods combined with cell lineage tracing techniques, we showed that deletion of $\beta$-catenin by either Acan-Cre ${ }^{\mathrm{ERT} 2}$ or CollOal-Cre resulted in greatly reduced cell trans-differentiation with a significant decrease in subchondral bone volume during mandibular condylar growth. Molecular studies demonstrated severe defects in cell proliferation and differentiation in both chondrocytes and bone cells. The gain of function studies (constitutive activation of $\beta$-catenin with Acan-Cre ${ }^{\mathrm{ERT}}$ at ages of postnatal day 7, 4-weeks and 6-months) led to more bone cell trans-differentiation of chondrocytes in the mandibular condyle due to increased proliferation and accelerated chondrocyte differentiation with incipient osteogenic changes within the cartilage matrix, resulting in an increased volume of poorly-formed immature subchondral bone. These results support the notion that chondrogenesis and osteogenesis are one continuous process, in which $\beta$-catenin signaling plays an essential role in the cell trans-differentiation of chondrocytes into bone cells during mandibular condylar development and growth.
\end{abstract}

Key words: chondrocyte, osteoblast, cartilage, cell signaling, growth/development

\section{Introduction}

The development and growth of the temporomandibular joint (TMJ) have long been recognized as different from that of long bone joints $[1,2]$. However, whether these cartilages with different developmental histories and structures also differ in their genetic regulation has only begun to be investigated in the last decade [3]. We have shown that deletion of Bmpr1a, a primary receptor for BMP-2 and BMP-4, in growth plate [4] and mandibular condylar cartilage (MCC) chondrocytes [5] not only leads to defects in chondrogenesis, but also in osteogenesis. These results support the new concept recently demonstrated by our lab and others that chondrocytes directly transform in considerable numbers into bone cells, thus making them the primary progenitor cells for endochondral bone formation rather than in-migrating cells from either bone marrow or periosteum [6-11]. In the growth 
plate, Bmpr1a null mice demonstrate a virtual cessation of endochondral bone formation [4] while in MCC of the same Bmpr1a null mice the subchondral bone phenotype is completely different [5]. This indicates that there may be diverse mechanisms for the same receptor in different skeletal locations (i.e., MCC vs growth plate) or that other regulators of chondrogenesis might affect MCC growth in unique ways.

Signaling via the $\mathrm{Wnt} / \beta$-catenin pathway is a well-established regulator of skeletal development and growth including growth plate and articular cartilage [12-20]. Cartilage-derived $\beta$-catenin is a central mediator for major events during endochondral bone formation, including chondrogenesis, primary and secondary ossification center development, vascularization, and perichondrial bone formation [14]. Moreover, $\beta$-catenin signaling is required for determining osteoblast versus chondrocyte cell fate and promoting chondrocyte proliferation and maturation [20]. In vivo mouse genetic studies using a constituently active form of $\beta$-catenin under the control of a Col 2 promoter demonstrated that the growth plate in postnatal mice undergoes closure within weeks of tamoxifen (TM) activation of the transgene [21]. $\beta$-catenin signaling in chondrocytes also plays a key role in the postnatal bone growth and bone remodeling likely through its regulation of osteoclast formation [22].

Most studies have focused on limb cartilages, with little attention to cartilages in the craniofacial region. Mice lacking $\mathrm{Wnt} / \beta$-catenin or with constitutive activation of $\mathrm{Wnt} / \beta$-catenin have been shown to exhibit disrupted growth in the cranial base synchondroses [23], but these are primary cartilages with developmental affinities to limb growth plate. The role of $\beta$-catenin signaling during TMJ development and growth has been little studied, with only two very different studies comprising our knowledge base; a developmental study showing agenesis of the MCC in mice with stabilization of $\beta$-catenin [24] and osteoarthritic changes in mice with conditional activation of $\beta$-catenin in MCC chondrocytes [25].

In this study, we employed recent advances in cell lineage tracing technology to investigate the role of $\beta$-catenin signaling in the regulation of condylar growth. Using the background of $R 26 R^{\text {Tomato, we }}$ demonstrated an essential role for $\beta$-catenin in the cell transformation of chondrocytes into bone cells (osteoblasts/osteocytes) by employing chondrocytespecific $\beta$-catenin loss-of-function models (using the $\beta$-catenin fx/fx line crossed to either the Aggrecan-Cre ${ }^{\text {ERT2 }}$ or Col10a1-Cre line) and gain-of-function genetic mouse models (using the tamoxifen-inducible Aggrecan-Cre ${ }^{\mathrm{ERT}}$ ).

\section{Materials and Methods}

\section{Breeding transgenic mice}

To generate triple mice to conditionally knockout $\beta$-catenin in all chondrocytes or specifically in hypertrophic chondrocytes, $\beta$-catenin ${ }^{\text {flox }}[26]$, and $R 26 R^{\text {Tomato }}$

(B6;129S6-Gt(ROSA)26Sortm9(CAG-tdTomato)Hze/J, stock number: 007905 from the Jackson Laboratory) were internally crossed with Aggrecan (Acan)-Cre ${ }^{\text {ERT2 }}$ (one-time tamoxifen injection at day 3, i.p.) [27] or Col10a1-Cre mice [28], respectively. Similarly, to generate triple mice for studies of the fate of chondrocytes with constitutively active $\beta$-catenin in chondrocytes (CA- $\beta$-cat) [29], Acan-Cre ${ }^{\mathrm{ERT} 2}$ (one-time tamoxifen injection at day 3 or 14, i.p.), $\beta$-catenin $f l o x(E x 3) / f l o x(E x 3)$, and $R 26 R^{\text {Tomato }}$ were internally crossed three times. Tamoxifen (Sigma, T5648) was dissolved in $90 \%$ corn oil (Sigma, C8267) and 10\% ethanol. Four animals were used in either control or mutant group at each time point.

All protocols were reviewed and approved by the Institutional Animal Care and Use Committee (IACUC) at Texas A\&M University College of Dentistry.

\section{Histological analysis}

Mandibular condyles were fixed in $4 \%$ paraformaldehyde and decalcified at $4{ }^{\circ} \mathrm{C}$. Samples for cell lineage tracing were dehydrated with sucrose and embedded in OCT followed by CryoJane frozen sections as previously described [30]. Samples for histological staining were embedded in paraffin, sectioned, and stained with safranin $\mathrm{O}$ (proteoglycans) or toluidine blue stain [31]. Immunostaining were proceeded as previously described [32] with the following antibodies: rabbit anti-aggrecan antibody (Abcam; 1:400), anti-collagen II mouse monoclonal antibody (Santa Cruz Biotechnology; 1:50), rabbit anti-collagen $X$ antibody (Abcam; 1:400), rabbit anti-DMP1 antibody (provided by Dr. Chunlin Qin at Texas A\&M University, 1:400), rabbit anti-Runx2 antibody (Abcam; 1:200), goat anti-sclerostin antibody (R\&D; 1:400), rabbit anti-collagen I antibody (Abcam; 1:100), anti-osteopontin mouse monoclonal antibody (Santa Cruz Biotechnology; $1: 100)$, or anti- $\beta$-catenin mouse monoclonal antibody (DSHB; 1:100). The immunohistochemistry experiments were detected with a 3, 3-diaminobenzidine kit (Vector Laboratories, Burlingame, CA). The immunofluorescent signals were detected with corresponding Alexa second 
antibody (Thermofisher, 1:200) at room temperature for 2 hours.

\section{Cell proliferation}

BrdU (Sigma, $10 \mathrm{mg} / \mathrm{ml}$ ) or EdU (Life technique, $10 \mathrm{mg} / \mathrm{ml}$ ) was injected into mice (two times for BrdU: 24- and 2 hours before sacrifice, i.p.; one time for EdU, 2 hours before sacrifice, i.p.). Image J was used to quantify the BrdU ${ }^{+}$or EdU ${ }^{+}$cells in MCC proliferating layers [5].

\section{Confocal microscopy}

SP5 Leica confocal microscope was used to capture the fluorescent cell images. All images were obtained at wavelengths ranging from 488 (green)-561 (red) $\mu \mathrm{m}$. Multiple stacked images were taken at $100 \mathrm{~Hz}$ and dimension of $1024 \times 1024$ [33]. Red color reflected by tomato signal indicated the descendant cells with Cre-activity; green color represented the corresponding immunofluorescent staining; blue color was DAPI staining.

\section{Radiographs}

The mandibles were dissected free of muscles and were taken X-ray by using a Faxitron model MX-20 Specimen Radiography System (Faxitron X-Ray Corp., Lincolnshire, IL, USA). The mandibular length was measured from the middle of the condylar head curvature to the most anterior point on the incisal alveolar process, and quantified by Image J software (Figure S2A) [5].

\section{Backscattered scanning electron microscopy (SEM), acid-etched SEM}

The mandibles were fixed in $4 \%$ paraformaldehyde, dehydrated in ascending concentrations of ethanol (from $70 \%$ to $100 \%$ ), and embedded in methyl-methacrylate (MMA, Buehler, Lake Bluff, IL) [34], which were cut and polished by using $1 \mu \mathrm{m}$ and $0.3 \mu \mathrm{m}$ alumina alpha micropolish II solution (Buehler). For acid etched SEM, samples were treated with $37 \%$ phosphoric acid for 2 to 10 seconds, $5 \%$ sodium hydrochloride for 5 minutes and then coated with gold (for acid-etched SEM imaging). For backscattered SEM, samples were coated with carbon. FEI/Philips XL30 field-emission environmental SEM (Hillsboro, OR, USA) was used to scan as described previously [35].

\section{Statistical Analysis}

All data were reported as mean (SD). The Kruskal-Wallis test was used to detect the significant differences among samples. The Mann-Whitney U test (post hoc test) was used to compare differences between the mutant and age-matched control groups. Significance level was defined as ${ }^{*} p<0.05 ;{ }^{* *} p<0.01$.

\section{Results}

Deleting $\beta$-catenin in early chondrocytes led to a malformed ramus, defective chondrogenesis, and a lack of trans-differentiation of chondrocytes into bone cells.

To address the impact of loss of $\beta$-catenin signaling (a cross of $\beta$-cateninfx/fx and Acan-Cre ${ }^{\text {ERT2 }}$ mice) on MCC and ramus formation, we induced the deletion of $\beta$-catenin event in the cartilage at P3 and harvested mice at ages of 2-, 4- and 12-weeks. X-ray images displayed a radiolucent area (correlated to the calcified cartilage region) in the 2 week-old condylar head with a low mineral density in the TMJ ramus (Figure 1A, upper right). By the ages of 4- and 12-weeks, a lack of calcified cartilage in the condylar head, an expanded ramus neck and a sharply reduced ramus length were evident (Figure 1A, middle and lower right panels). Mandibular length was significantly reduced compared to controls at the age of 12-weeks (Figure 1B). Toluidine blue-stained sections of 2-week-old cKO condyles showed a reduction in the metachromatic area of the $\mathrm{MCC}$, largely due to the virtual absence of the region of flattened and early chondrocytes (Figure 1C). Importantly, there was essentially no trabecular bone present except immediately deep to the MCC in cKO mice. The EdU assay showed a significant reduction in cell proliferation in the prechondroblastic zone of the cKO MCC compared to controls (Figure 1D; white arrow).

To trace the cell fate of chondrocytes lacking $\beta$-catenin signaling, we generated the above cKO mice in the background of $R 26 R^{\text {Tomato }}$ (i.e., deletion of $\beta$-catenin and activation of red tomato in chondrocytes occurred at 3 days of age and traced the cell fate at 14 days). The confocal images displayed the expected presence of numerous red cells in the subchondral bone, reflecting their initial cell origin as chondrocytes. When combined with green IHC signals, Col2 (Figure 1E), Col 10 (Figure 1F) or DMP1 (Figure 1G; osteocyte marker) were located as expected in the control group (left panels). However, in cKO mice, there were few red bone cells in the area deep to the immediate subchondral bone, resulting in an open region within the ramus (Figure 1E-G). Furthermore, the extent of immunoreactivity for $\mathrm{Col} 2$ and $\mathrm{Col} 10$ was greatly reduced compared to controls (Figure 1E-F). DMP1 signal was greatly reduced in the trabecular bone of cKO mice (Figure 1G). In 4-week old cKO mice (induced at day 3), Col 10 expression was largely undetectable (Figure S1A). The combined red tomato and green DMP1 images revealed few transformed red bone cells in the subchondral bone area (Figure S1B). 


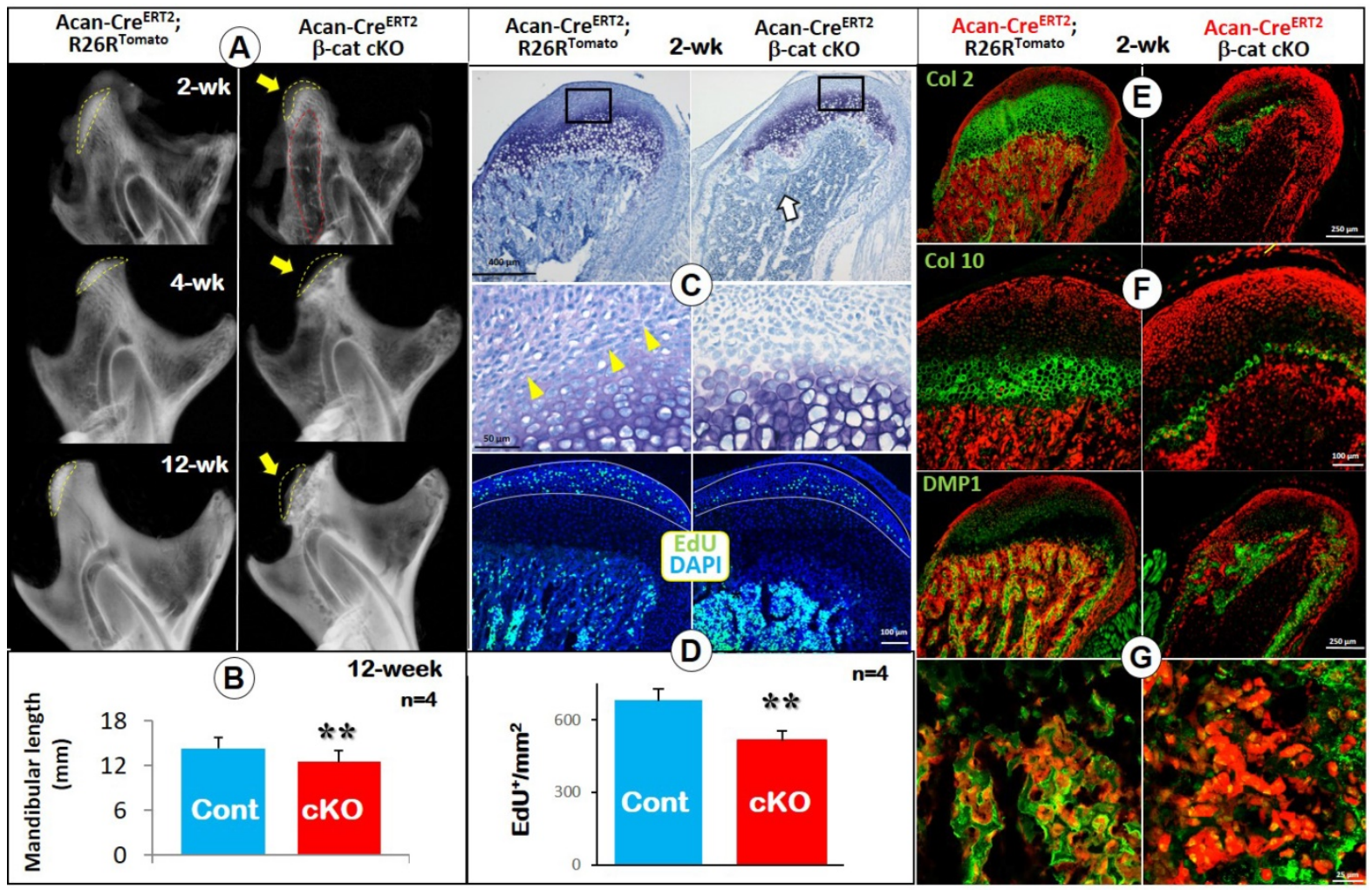

Figure 1. Deletion of $\beta$-catenin in early chondrocytes led to malformed condylar neck, defective chondrogenesis, and reduced chondrocyte transformation. (A) X-ray images displayed a lack of calcified cartilage region (yellow dotted line) on mutant mice (arrow in upper panel) with a low mineralized condylar ramus (red dotted line) in 2-week old Acan-CreERT2; $\beta$-catenin CKO mice, and a small condylar head formation (arrows in middle and lower panel), an expanded ramus neck and a sharp reduction in the ramus length at ages of 4- and 12-weeks. (B) Quantitative data of mandibular length revealed a statistically significant reduction of the mandibular length in 12-week old cKO mice $(n=4)$. (C) Toluidine blue staining for 2-week old cKO condyles showed a reduction in the metachromatic area of the MCC due to decreased flattened chondrocytes (yellow arrows), and a lack of subchondral bone (white arrow). (D) EdU + cells were significantly reduced in proliferating zone of the CKO MCC $(n=4)$. (E) Col 2 IHC combined with cell lineage tracing (Acan-CreERT2 was activated at P3, reflecting the initial cell origin in chondrocytes and its transformed bone cells) showed a sharp decrease of Col $2^{+}$area in cKO MCC. (F) Col $10 \mathrm{IHC}$ demonstrated a dramatic reduction in Col 10-expressing cells in cKO MCC, indicating a defective chondrocyte hypertrophy. (G) DMPI IHC confirmed the dramatic reduction of subchondral bone volume, with less mature osteocytes expressing DMPI in CKO mice (high magnification in the lower panel).

Together, the above studies not only confirmed a critical role for $\beta$-catenin signaling in MCC chondrogenesis but also revealed a novel role for $\beta$-catenin in controlling cell trans-differentiation of chondrocytes into bone cells during MCC growth.

\section{Deletion of $\beta$-catenin in hypertrophic chondrocytes (HCs) resulted in diminished endochondral bone formation.}

Although Acan-Cre ${ }^{\text {ERT2 }}$ line is expressed in all chondrocytes, the deletion of $\beta$-catenin by this Cre mainly reflects the impact of $\beta$-catenin in early chondrocytes (Figure 1 ). To test the role of $\beta$-catenin in the cell trans-differentiation from HCs into bone cells, we generated a compound mouse line containing Col10a1-Cre, $\beta$-catenin $f x / f x$ and $R 26 R^{\text {Tomato }}$ and harvested the mice at age of 2 weeks. X-ray images showed an expanded radiolucent area (correlated to the calcified cartilage region) in the 2 week-old condylar head with an extremely low mineral density in the MCC ramus (Figure 2A). Toluidine blue-stained sections displayed an expansion of all MCC cartilage layers with a particular increase in the $\mathrm{CKO} \mathrm{HCs}$ (Figure 2B). Similar to the phenotype in Acan-Cre ${ }^{\text {ERT2; }}$ $\beta$-catenin cKO mice, there was no trabecular bone present (Figure 2A-B). Cell lineage studies combined with IHC demonstrated a considerably thickened Col $2^{+}$region (Figure $2 \mathrm{C}$ ), as well as a somewhat increased thickness of $\mathrm{Col} 10^{+}$cells (Figure 2D) in the cKO mice. In the thin layer of cKO subchondral bone, there were sharp reductions in expression of Runx2 (a marker of preosteoblasts; Figure 2E) and two markers of osteocytes: SOST (Figure 2F) and DMP1 (Figure 2G), indicating an additional role for $\beta$-catenin in the continuing bone cell maturation after the cell trans-differentiation.

Taken together, use of both Acan-Cre ${ }^{\text {ERT2 }}$ and Col10a1-Cre loss-of-function models demonstrated that $\beta$-catenin plays a critical role in MCC proliferation and chondrogenesis and in the trans-differentiation of chondrocytes into bone cells, the major source of bone cells during MCC endochondral bone formation. 


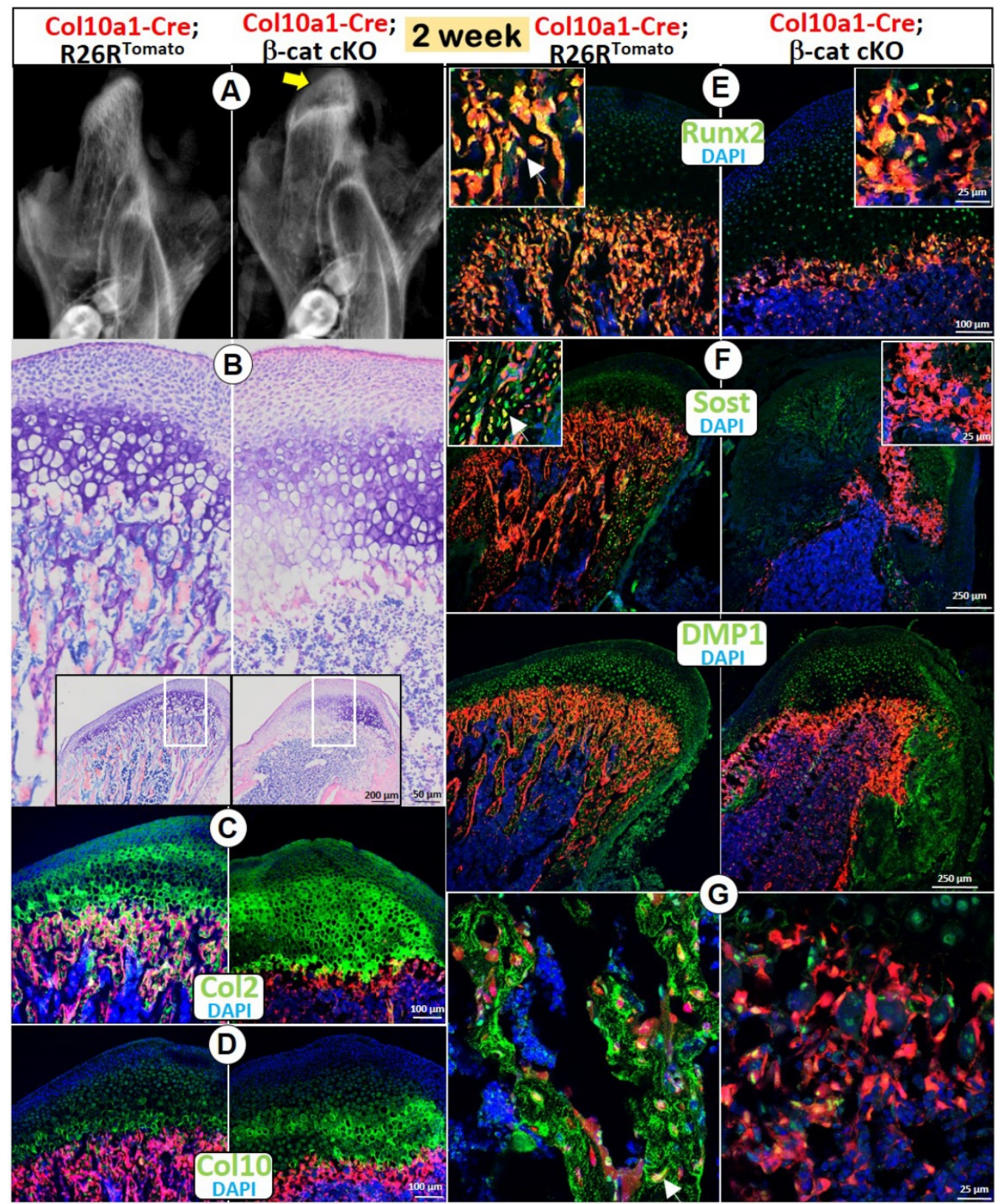

Figure 2. Deletion of $\boldsymbol{\beta}$-catenin in HCs resulted in diminished endochondral bone formation. (A) The $X$-ray images showed an expanded radiolucent area (correlated to the calcified cartilage region, yellow arrow) with an extremely low mineralized ramus in the 2 -week old CollOal-Cre; $\beta$-catenin cKO mice. (B) Toluidine blue staining displayed an expansion of all MCC cartilage layers in cKO mice with a particular increase in HCs, but with no trabecular bone present in subchondral bone area. (C) Col 2 IHC combined with cell lineage tracing (CollOal-Cre began activation at E14.5, in HCs and their transformed bone cells) demonstrated a considerably thickened Col2+ region in cKO mice. (D) Coll $0^{+}$cells increased in cKO compared with control mice. (E-G) There was only a thin layer of cKO subchondral bone in cKO mice, with a sharp reduction in expression of Runx2 (E), SOST (F) and DMP1 (G) compared with the control mice (arrows).

\section{Constitutive activation of $\beta$-catenin in MCC accelerated chondrogenesis and cell trans-differentiation coupled with widespread calcification in the cartilage}

To further address the role of $\beta$-catenin in MCC growth, we generated a gain-of-function model containing Acan-Cre ${ }^{E R T 2}, \quad \beta$-cateninflox(Ex3)fflox(Ex3) and $R 26 R^{\text {Tomato }}$ (CA- $\beta$-cat mice), and constitutively activated $\beta$-catenin in chondrocytes for 5 days (one-time tamoxifen injection at day 3 and harvested at day 7); 14 days (one-time tamoxifen injection at 2-week and harvested at 4-week); 5.5 months (one-time tamoxifen injection at 2-week and harvested at 6-month), respectively.

The early activation of $\beta$-catenin in chondrocytes greatly enhanced chondrogenesis reflected by sharp increases in Col 2 expression (Figure $3 \mathrm{~A}$ ), and osteogenesis revealed by increases in Col 1 (Figure 3B) and DMP1 expression (Figure 3C). Importantly, there 
was more accelerated trans-differentiation of chondrocytes into red bone cells in the cartilage region of CA- $\beta$-cat mice than in wild type mice (Figure 3, white arrows).

The activation of $\beta$-catenin in chondrocytes after the initial MCC pattern formation generated a similar phenotype (induced at 2 weeks, harvested at 4 weeks). In X-ray images, the mandibular condyle was more highly mineralized (Figure 4A), but the length of condylar process was statistically shorter than normal in the CA- $\beta$-cat mice (Figure S2B). Back-scattered SEM (Figure 4B, central) and acid-etched SEM (Figure 4B, lateral) demonstrated that the calcified $\mathrm{HC}$ layer in the mutant MCC was thicker with many more HCs

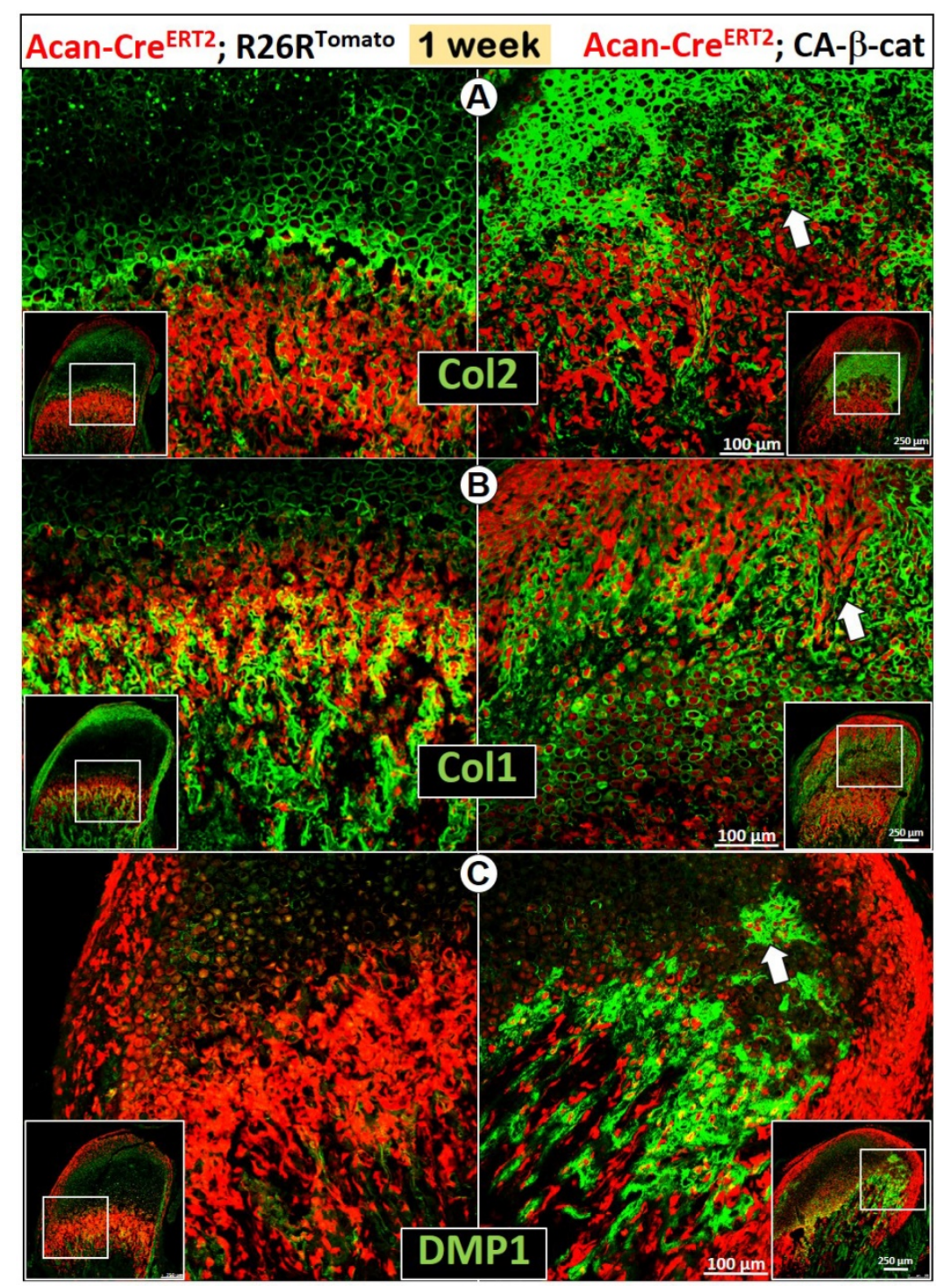

Figure 3. Constitutive activation of $\beta$-catenin in MCC accelerated chondrogenesis and osteogenesis in early postnatal development. (A) By using Acan-CreERT2; $\beta$-cateninflox $(\mathrm{Ex} 3)$ ffox $(\mathrm{E} \times 3)$ mice with $\mathrm{Cre}$ activation at $\mathrm{P} 3$, we found that $\mathrm{Col} 2 \mathrm{IHC}$ was more intense in CA- $\beta$-cat mice at P7 (arrow). (B-C) High level of Col 1 (B) and DMPI (C) expression in 7-day CA- $\beta$-cat mice indicated the increased osteogenesis in MCC. In addition, there was more accelerated trans-differentiation of chondrocytes into red bone cells in the cartilage region of CA- $\beta$-cat mice (arrows). than control, which was further confirmed by widespread Col 10 expression in CA- $\beta$-cat mice (Figure S2C). Goldner staining (Figure S2D) also suggested that MCC in CA- $\beta$-cat mice was more widely mineralized (indicated by green color).

BrdU positive cells in MCC were significantly increased in CA- $\beta$-cat mice (Figure $4 \mathrm{C}$ ). The total thickness of the CA- $\beta$-cat MCC was greater than in controls, but with disruptions in the normal cell layering such that HCs were present throughout the depth of the tissue but with much fainter Safranin O staining (Figure 4D). IHC against osteopontin (OPN, a marker for early osteogenic cells) demonstrated dramatically expanded OPN expression in the CA- $\beta$-cat MCC (Figure 4E), indicating a transition to a more osteogenic phenotype.

To further investigate the cellular trans-differentiation, we performed cell lineage tracing, which revealed more and enlarged early chondrocytes in the superficial aspect of the CA- $\beta$-cat MCC, indicating an acceleration of chondrogenesis starting from the early stage (Figure 5). Co-IHC staining with Col 2 (Figure 5A) and Col 10 (Figure S2C) demonstrated more discontinuous and patchy immunoreactivity in the CA- $\beta$-cat MCC. Strikingly, the flattened chondrocytes and HCs pericellularly expressed high levels of bone markers of Col 1 (Figure 5B) and DMP1 (Figure $5 \mathrm{C})$, supporting the accelerated transdifferentiation. Interestingly, there were many red cells, which varied in size, that did not express either cartilage or bone markers (Figure S3; arrowheads), indicating a lack of cell activity.

By the age of 6 months, the CA- $\beta$-cat mice displayed a similar but more severe phenotype. X-ray images showed a short but hypermineralized mandibular condyle in the CA- $\beta$-cat mice (Figure S4A), and the length of mandible was significantly shorter in the mutant mice (Figure S4B). The back scattered SEM image showed a wider condyle with uneven mineralization in the CA- $\beta$-cat condylar neck (Figure $\mathrm{S} 4 \mathrm{C})$, Both toluidine blue staining (Figure S4D) and Col 2 IHC (Figure S4E) revealed expanded hypertrophic chondrocytes present throughout the mutant MCC. 

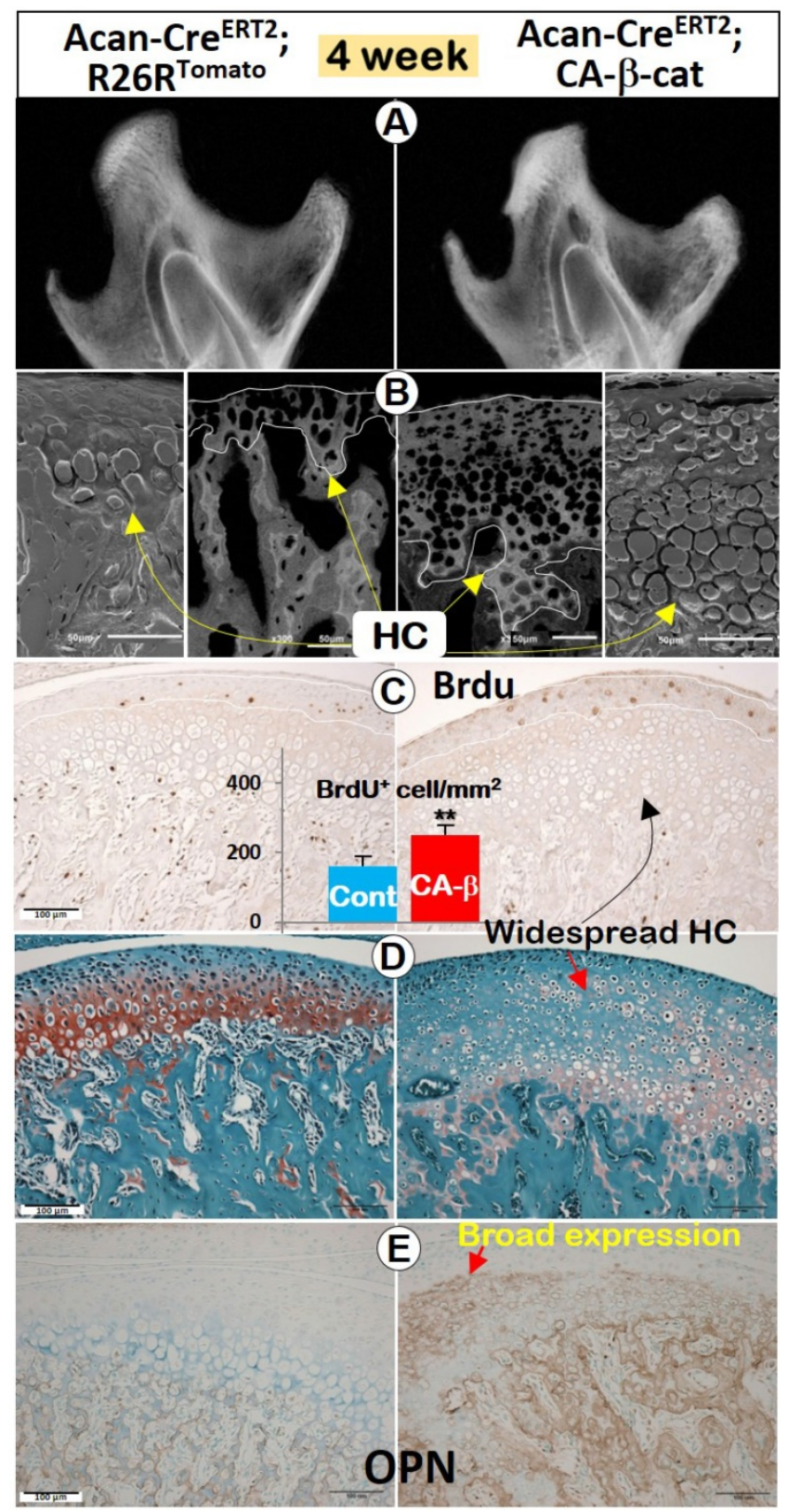

Figure 4. Stabilization of $\beta$-catenin in 4-week old mice enhanced chondrogenesis, promoted chondrocyte proliferation, and affected condylar ramus formation. (A) $X$-ray images showed that the CA- $\beta$-cat mandibular condyle was highly mineralized with a significantly shorter mandibular length (Cre was activated at 2 weeks, $n=4$ ). (B) Back-scattered SEM (central) and acid-etched SEM (lateral) demonstrated that the calcified $\mathrm{HC}$ layer in the mutant MCC was thicker with many more hypertrophic chondrocytes than control (yellow arrows). (C) BrdU' cells in the proliferating zone of mutant mice were significantly increased, indicating higher cell proliferation $(n=4)$. (D) Thickness of Safranin O staining was greater in CA- $\beta$-cat MCC than in controls, but with disruption of the normal cell layering. (E) OPN expression was dramatically expanded in the CA- $\beta$-cat MCC, indicating that the ossification of chondrocytes was accelerated.

In summary, our studies at three different ages demonstrate that constitutive activation of $\beta$-catenin promotes acceleration of chondrogenesis, osteogenesis and cell trans-differentiation during MCC growth.

\section{Discussion}

For many years, chondrogenesis and osteogenesis were thought to be closely linked but separate processes during endochondral bone formation [36]. However, the studies from other groups [8-11] and our recent report in long bone challenged this dogma and raised a new hypothesis: chondrogenesis (phase one) and osteogenesis (phase two) are not separate processes but one continuous biological process [37]. In this study, our loss- and gain-of-function models of $\beta$-catenin at different developmental stages demonstrated that $\beta$-catenin is a key regulator of proliferation and chondrocyte differentiation as well as the direct trans-differentiation of chondrocytes into bone cells during postnatal MCC growth (Figure 5D). The deletion of $\beta$-catenin in aggrecan-secreting cells (Figure 1) or HCs (Figure 2) produced a similar phenotype: disruptions of chondrogenesis (especially cell differentiation), a sharp reduction in mandibular length, and a drastic reduction in trans-differentiation of chondrocytes into bone cells, as well as bone cell maturation. By contrast, constitutive activation of $\beta$-catenin in chondrocytes at three ages [P3 to P7 (Figure 3), P14 to 1-month (Figure 4-5), and P14 to 6-months (Figure S4)] greatly accelerated chondrogenesis, and the trans-differentiation of chondrocytes into bone cells, leading to a shorter but wider condylar neck.

Deletion of $\beta$-catenin by Acan-Cre ${ }^{\mathrm{ERT} 2}$ affects both cell proliferation and cell differentiation in all chondrocytes, whereas deletion of $\beta$-catenin by Col10a1-Cre mainly induces failure of HCs to trans-differentiate into bone cells, leading to a great accumulation of $\mathrm{HCs}$ and pre-hypertrophic chondrocytes. However, both cKO mice models display almost identical subchondral bone phenotypes: a sharp reduction in bone volume, a great decrease in cell trans-differentiation, and a defect in cell maturation (as reflected by bone markers). This finding argues strongly that MCC subchondral bone is mainly formed via cell trans-differentiation from HCs (instead of early chondrocytes) under regulation by $\beta$-catenin, which is in agreement with previous studies in long bone [17, 37].

It is well documented that the cKO event is not a complete gene deletion process due to the limitation of Cre efficiency [38], which explains why some red bone cells and subchondral bone are still present in cKO mice (Figure 1-2). Currently, we do not know why bone markers are largely undetected in the chondrocyte-derived bone cells in both cKO models (i.e., immature bone cells, Figures 1-2) as neither Cre 
line is active in bone cells. We reason that $\beta$-catenin is not only important for cell trans-differentiation but also critical for bone marker expression even before the cell trans-differentiation event, as we showed that Col 1 and DMP1 are expressed in HCs (Figure S5). In other words, osteogenic genes are already activated in HCs. In fact, bone markers expressed in HCs have been reported in the literature [39]. Of note, the subchondral bone phenotype is more severe in the Col10a1 cKO mice, which is likely due to its

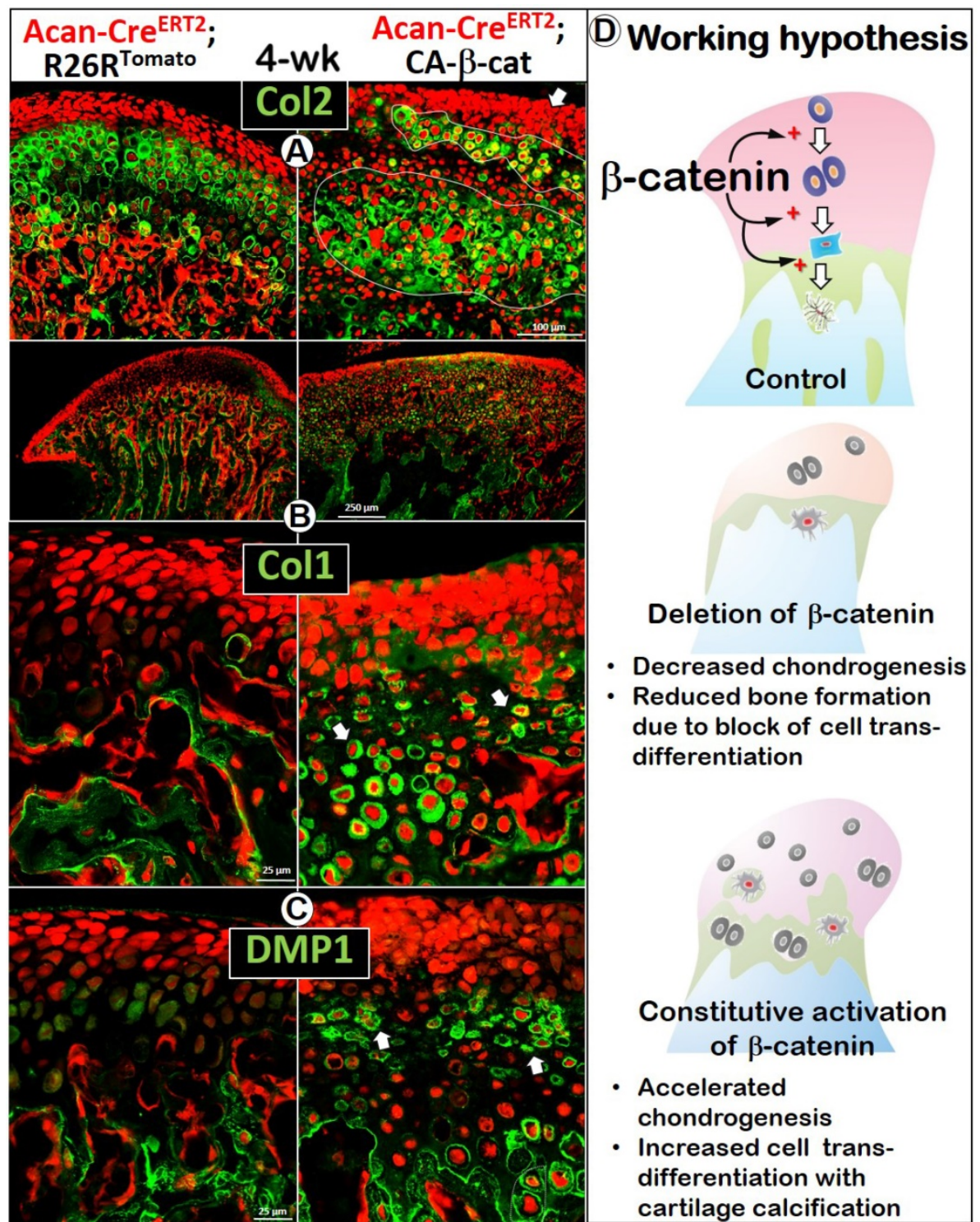

Figure 5. Constitutive activation of $\beta$-catenin led to accelerated cell-transformation from chondrocytes into bone cells, giving rise to osteogenesis in the cartilage. (A) Co-IHC staining with Col 2 in the CA- $\beta$-cat MCC displayed a more widespread, denser, but patchy area of chondrocytes, with many more red prechondroblastic cells (arrow) near the MCC surface. (B) Col 1 expression was much higher (arrows) in CA- $\beta$-cat MCC (high magnification in lower panel). (C) Many chondrocytes also expressed high level of DMP1, reflecting the accelerated trans-differentiation from chondrocytes into bone cells in CA- $\beta$-cat MCC (arrows). (D) Using both conditional deletion and constitutive activation of $\beta$-catenin, our study demonstrates the critical role of $\beta$-catenin in the direct trans-differentiation of chondrocytes to bone cells during MCC growth. The lack of $\beta$-catenin led to a decrease of chondrogenesis and chondrocyte-derived bone cells, accompanied by reduction of subchondral bone volume; the stabilization of $\beta$-catenin gave rise to more chondrogenesis and cell trans-differentiation, along with cartilage calcification in MCC. cumulative effects beginning at E14.5 [6].

Although the gain of function model supports the role of $\beta$-catenin in the cell trans-differentiation hypothesis, the mutant ramus length is shorter than controls. We reason that the following two factors are likely responsible for this unexpected phenotype: a. the prematurely formed bone cells do not have the full function of mature bone cells, and the existing cartilage matrix may interfere with bone matrix production; $\mathbf{b}$. the pattern of MCC ramus is largely formed by the time that $\beta$-catenin is constitutively activated and the existing bone matrix "blockades" the newly formed bone.

The pronounced changes in MCC gene expression profiles and structure in both mutant $\mathrm{cKO}$ models are in agreement with previous studies showing delayed chondrocyte hypertrophy and reduced proliferation in mouse growth plates in which $\beta$-catenin was deleted in chondrocytes and perichondrial cells [18-20]. The phenotype in the MCC of the CA- $\beta$-cat mice is even more striking, presenting a picture of a cartilage transitioning to bone (reduced Safranin O, immunoreactivity for $\mathrm{Col} 1$, osteopontin and DMP1 in the cartilage). This phenotype is similar in some respects to the only other study of the TMJ in mice with constitutively activated $\beta$-catenin, which reported progressive decreases in Alcian blue and Col 2 staining in 1-6 month-old CA- $\beta$-cat mice as well as increased Col 10 staining and greater numbers of hypertrophic chondrocytes that they interpreted as evidence of accelerated chondrocyte hypertrophy [25]. Thus, $\beta$-catenin appears to have a similar regulatory effect on both long bone and TMJ. Currently, our group is searching for other genes that regulate the unique growth of the MCC. 


\section{Supplementary Material}

Supplementary figures.

http://www.ijbs.com/v14p0001s1.pdf

\section{Acknowledgment}

This study was partially supported by National Institutes of Health: DE025659 to JQF; DE024797 to SHE; National Natural Science Foundation of China (81500876) to JJJ.

\section{Competing Interests}

The authors have declared that no competing interest exists.

\section{References}

1. Merida-Velasco JR, Rodriguez-Vazquez JF, Merida-Velasco JA, Sanchez-Montesinos I, Espin-Ferra J, Jimenez-Collado J. Development of the human temporomandibular joint. Anat Rec. 1999; 255: 20-33.

2. Shibata S, Suda N, Suzuki S, Fukuoka H, Yamashita Y. An in situ hybridization study of Runx2, Osterix, and Sox9 at the onset of condylar cartilage formation in fetal mouse mandible. J Anat. 2006; 208: 169-77.

3. Hinton RJ. Genes that regulate morphogenesis and growth of the temporomandibular joint: a review. Dev Dyn. 2014; 243: 864-74.

4. Jing J, Ren Y, Zong Z, Liu C, Kamiya N, Mishina Y, et al. BMP receptor 1A determines the cell fate of the postnatal growth plate. Int J Biol Sci. 2013; 9: 895-906.

5. Jing J, Hinton RJ, Mishina Y, Liu Y, Zhou X, Feng JQ. Critical role of Bmpr1a in mandibular condyle growth. Connect Tissue Res. 2014; 55 Suppl 1: 73-8.

6. Jing Y, Zhou X, Han X, Jing J, von der Mark K, Wang J, et al. Chondrocytes Directly Transform into Bone Cells in Mandibular Condyle Growth. J Dent Res. 2015; 94: 1668-75.

7. Hinton RJ, Jing Y, Jing J, Feng JQ. Roles of Chondrocytes in Endochondral Bone Formation and Fracture Repair. J Dent Res. 2017; 96: 23-30.

8. Zhou X, von der Mark K, Henry S, Norton W, Adams H, de Crombrugghe B. Chondrocytes transdifferentiate into osteoblasts in endochondral bone during development, postnatal growth and fracture healing in mice. PLoS Genet. 2014; 10: e1004820.

9. Yang G, Zhu L, Hou N, Lan Y, Wu XM, Zhou B, et al. Osteogenic fate of hypertrophic chondrocytes. Cell Res. 2014; 24: 1266-9.

10. Yang L, Tsang KY, Tang HC, Chan D, Cheah KS. Hypertrophic chondrocytes can become osteoblasts and osteocytes in endochondral bone formation. Proc Natl Acad Sci U S A. 2014; 111: 12097-102.

11. Park J, Gebhardt M, Golovchenko S, Perez-Branguli F, Hattori T, Hartmann C, et al. Dual pathways to endochondral osteoblasts: a novel chondrocyte-derived osteoprogenitor cell identified in hypertrophic cartilage. Biol Open. 2015; 4: 608-21.

12. Hartmann C, Tabin CJ. Dual roles of Wnt signaling during chondrogenesis in the chicken limb. Development. 2000; 127: 3141-59.

13. Yasuhara R, Ohta Y, Yuasa T, Kondo N, Hoang T, Addya S, et al. Roles of beta-catenin signaling in phenotypic expression and proliferation of articular cartilage superficial zone cells. Lab Invest. 2011; 91: 1739-52.

14. Dao DY, Jonason JH, Zhang $\mathrm{Y}$, Hsu W, Chen D, Hilton MJ, et al Cartilage-specific beta-catenin signaling regulates chondrocyte maturation, generation of ossification centers, and perichondrial bone formation during skeletal development. J Bone Miner Res. 2012; 27: 1680-94.

15. Golovchenko S, Hattori T, Hartmann C, Gebhardt M, Gebhard S, Hess A, et al. Deletion of beta catenin in hypertrophic growth plate chondrocytes impairs trabecular bone formation. Bone. 2013; 55: 102-12.

16. Usami $Y$, Gunawardena AT, Iwamoto M, Enomoto-Iwamoto M. Wnt signaling in cartilage development and diseases: lessons from animal studies. Lab Invest. 2016; 96: 186-96.

17. Houben A, Kostanova-Poliakova D, Weissenbock M, Graf J, Teufel S, von der Mark K, et al. beta-catenin activity in late hypertrophic chondrocytes locally orchestrates osteoblastogenesis and osteoclastogenesis. Development. 2016; 143: 3826-38.

18. Hill TP, Spater D, Taketo MM, Birchmeier W, Hartmann C. Canonical Wnt/beta-catenin signaling prevents osteoblasts from differentiating into chondrocytes. Dev Cell. 2005; 8: 727-38.

19. Hu H, Hilton MJ, Tu X, Yu K, Ornitz DM, Long F. Sequential roles of Hedgehog and Wnt signaling in osteoblast development. Development. 2005; 132: 49-60.

20. Day TF, Guo XZ, Garrett-Beal L, Yang YZ. Wnt/beta-catenin signaling in mesenchymal progenitors controls osteoblast and chondrocyte differentiation during vertebrate skeletogenesis. Dev Cell. 2005; 8: 739-50.

21. Yuasa T, Kondo N, Yasuhara R, Shimono K, Mackem S, Pacifici M, et al. Transient activation of Wnt/\{beta\}-catenin signaling induces abnormal growth plate closure and articular cartilage thickening in postnatal mice. Am J Pathol. 2009; 175: 1993-2003.

22. Wang B, Jin H, Zhu M, Li J, Zhao L, Zhang Y, et al. Chondrocyte beta-catenin signaling regulates postnatal bone remodeling through modulation of osteoclast formation in a murine model. Arthritis Rheumatol. 2014; 66: 107-20.

23. Nagayama M, Iwamoto M, Hargett A, Kamiya N, Tamamura Y, Young B, et al. Wnt/beta-catenin signaling regulates cranial base development and growth. J Dent Res. 2008; 87: 244-9.

24. Wang Y, Liu C, Rohr J, Liu H, He F, Yu J, et al. Tissue interaction is required for glenoid fossa development during temporomandibular joint formation. Dev Dyn. 2011; 240: 2466-73.

25. Wang M, Li S, Xie W, Shen J, Im HJ, Holz JD, et al. Activation of beta-catenin signalling leads to temporomandibular joint defects. Eur Cell Mater. 2014; 28: 223-35.

26. Brault V, Moore R, Kutsch S, Ishibashi M, Rowitch DH, McMahon AP, et al. Inactivation of the beta-catenin gene by Wnt1-Cre-mediated deletion results in dramatic brain malformation and failure of craniofacial development. Development. 2001; 128: 1253-64.

27. Henry SP, Jang CW, Deng JM, Zhang Z, Behringer RR, de Crombrugghe B. Generation of aggrecan-CreERT2 knockin mice for inducible Cre activity in adult cartilage. Genesis. 2009; 47: 805-14

28. Gebhard S, Hattori T, Bauer E, Schlund B, Bosl MR, de Crombrugghe B, et al. Specific expression of Cre recombinase in hypertrophic cartilage under the control of a BAC-Col10a1 promoter. Matrix Biol. 2008; 27: 693-9.

29. Harada N, Tamai Y, Ishikawa T, Sauer B, Takaku K, Oshima M, et al. Intestinal polyposis in mice with a dominant stable mutation of the beta-catenin gene. EMBO J. 1999; 18: 5931-42.

30. Jiang X, Kalajzic Z, Maye P, Braut A, Bellizzi J, Mina M, et al. Histological analysis of GFP expression in murine bone. J Histochem Cytochem. 2005; 53: 593-602.

31. Zhang R, Lu Y, Ye L, Yuan B, Yu S, Qin C, et al. Unique roles of phosphorus in endochondral bone formation and osteocyte maturation. J Bone Miner Res. 2011; 26: 1047-56

32. Jing Y, Hinton RJ, Chan KS, Feng JQ. Co-localization of Cell Lineage Markers and the Tomato Signal. J Vis Exp. 2016; $10.3791 / 54982$.

33. Ren Y, Lin S, Jing Y, Dechow PC, Feng JQ. A novel way to statistically analyze morphologic changes in Dmp1-null osteocytes. Connect Tissue Res. 2014; 55 Suppl 1: 129-33.

34. Feng JQ, Ward LM, Liu S, Lu Y, Xie Y, Yuan B, et al. Loss of DMP1 causes rickets and osteomalacia and identifies a role for osteocytes in mineral metabolism. Nat Genet. 2006; 38: 1310-5.

35. Lu Y, Yuan B, Qin C, Cao Z, Xie Y, Dallas SL, et al. The biological function of DMP-1 in osteocyte maturation is mediated by its $57-\mathrm{kDa}$ C-terminal fragment. J Bone Miner Res. 2011; 26: 331-40.

36. Ornitz DM, Marie PJ. FGF signaling pathways in endochondral and intramembranous bone development and human genetic disease. Genes Dev. 2002; 16: 1446-65.

37. Jing Y, Jing J, Ye L, Liu X, Harris SE, Hinton RJ, et al. Chondrogenesis and osteogenesis are one continuous developmental and lineage defined biological process. Sci Rep. 2017; 7: 10020.

38. Nagy A. Cre recombinase: the universal reagent for genome tailoring. Genesis. 2000; 26: 99-109.

39. Gerstenfeld LC, Shapiro FD. Expression of bone-specific genes by hypertrophic chondrocytes: implication of the complex functions of the hypertrophic chondrocyte during endochondral bone development. J Cell Biochem. 1996; 62: 1-9. 